\title{
The Electromagnetic Transducers of Asymmetry of Three-phases Electrical Currents to Voltage
}

\author{
Siddikov Ilkhomjon Khakimovich \\ Department of Power Supply Systems, Tashkent University of Information Technology, Uzbekistan
}

Copyright (C) 2015 by authors, all rights reserved. Authors agree that this article remains permanently open access under the terms of the Creative Commons Attribution License 4.0 International License

\begin{abstract}
Unbalanced currents and voltage of three-phases electrical nets are an important cause of asymmetric power power, and there are determinate of quality of electrical power. Three-phase electrical nets are balanced or symmetrical, when the voltage and current of each phases have a same amplitudes and phases. If we do not at least one of these conditions, the system is considered asymmetrical values and parameters. This article is devoted effectiveness of measures of asymmetry on the base of electromagnetic transducers of information about three-phases primary currents to signal as secondary voltage.
\end{abstract}

Keywords Current, Voltage, Transducers, Electromagnet

\section{Introduction}

During control of the processes of production, transmission, distribution and consumption of power determining importance is accuracy, sensitivity, linearity and commonality parameters of the elements end devices of control systems with primary facilities of transformation current, because inaccuracy of the control of power and parameters will accompany with significant negative economic damage. The development of the complex approach, providing power and resource saving on base of efficiently control sources of reactive power, expansion of the functional possibilities, simplification of designs, reduction of weight and volume factors, improvement of the technologies of fabrication, provision of the remote measuring processes, transformation of the current based on the usage of the modern primary measuring transducers is actual problem of the control devices and elements of power system. Herewith, the primary measuring current transducers, being the main section information-measuring and controlling systems, completely define the technical and economic factors of power system.

At present employment of the electromagnetic transducers of current with unified output value, expansion of the spectrum converted electric values is limited, because of the insufficient development of the methods of calculation and research of portioned magnetic systems of the transducers. The classical methods of research of the magnetic circuits do not provide necessary accuracy due to the asymmetries of the three-phase primary currents of electric network, which do not possess the sufficient generality, covering herewith only main varieties of the circuits of electric and magnetic nature. The magnetic systems of the transformation with on linear parameters and features of the primary current of electric network of power system during calculations has been taken as object with concentrated parameters [1].

\section{Asymmetry of the Currents of Three-phases Electrical Nets}

The Main factor of the appearances of inaccuracy of transducers of the current are: no symmetry of three-phases currents and voltages in values and phases, changing of the frequency, change of the temperature, appearance of the harmonicas currents and voltages of three-phases electrical nets, vibratory of loads when functioning the transducers and others. The System analysis of the electromagnetic transducers of the primary current has allowed also that classical designs of the transducers of the current managerial system by reactive power - a transformers of the current provide on output current by value $5 \mathrm{~A}$ under nominate of primary, have: restriction on range of the converted current; significant inaccuracy; complex and non-technological to designs; the greater size; the mass; specific consumption of materials and cost. Under nominate of input current, require the matching element - an additional transformer for unification of the joint work with modern microprocessors and controlling personal computer.

The Existing electromagnetic transducers of the current when controlling sources of reactive power of the power systems do not provide necessary accuracy of information 
about currents of the three-phase electric nets, because single-phase performance of the magnetic systems of the transformation have a nonlinearity of the saturation of the magnetic systems and non-commonality importance of the output values. This fact a limited using the classical electromagnetic transducers of the current in corresponding to managerial system of the values and parameter of the three-phases electric nets. So development, research of the electromagnetic transducers with flat measuring winding primary one and three-phase currents to secondary voltage with extended functional possibility and unified output value, taking into account no symmetry of three-phase current and creation on their base of the systems of multifunction control reactive power is a decision of the problem of the improvement and developments existing technologies of the checking and control value and parameter of electric current and voltage is important and actual problem of the power systems.

The purpose of research is the development of theories and designing the electromagnetic transducers one and multiphase's primary current to secondary voltage with flat measuring windings for multifunction control asymmetry currents of sources of power and creation of their base of power and recourse saving in power systems.

In single-phases electromagnetic transdusers of the current to voltage, got according of next equarion

$$
U_{\text {eout }}=4,44 f W F_{\max A} e^{-\frac{R_{\mathrm{I}} t}{L_{\mathrm{I}}}} \pm F_{r e m ~ A} e^{-\frac{R_{\mathrm{I}} t}{L_{\mathrm{I}}}}
$$

Similar formulas for building of the steady-state features for secondary voltages of phases $\mathrm{B}$ and $\mathrm{C}$ three-phase electric nets:

$$
\begin{gathered}
U_{\text {eout }}=4,44 f W F_{\max B} e^{-\frac{R_{\mathrm{II}} t}{L_{\mathrm{II}}}} \pm F_{\mathrm{rem} B} e^{-\frac{R_{\mathrm{II}} t}{L_{\mathrm{II}}}} \\
U_{\text {eout }}=4,44 f W F_{\max C} e^{-\frac{R_{\mathrm{III}} t}{L_{\mathrm{III}}}} \pm F_{\mathrm{rem} C} e^{-\frac{R_{\mathrm{III}} t}{L_{\mathrm{III}}}}
\end{gathered}
$$

Principle schemes of the electromagnetic transducers of the current to voltage with flat measure winding for combined auto control of source given in Figure 1 [2].

Designed schemes of the combined auto control of source of reactive power on base of the electromagnetic transducers of the current to voltage with flat measure winding practically not insertion, provide pinpoint accuracy and unification out signal universal when using in combined auto control system of steady-state and dynamic source of reactive power of power system, consume the small power, have not a rolling parts, differ high reliability.
The highest magnetic flows, vastly exceeding amplitude of importance formed flows, exist a remaining magnetic flows from different courses $-F_{\text {rem } A}, F_{\text {rem } B}, F_{\text {rem } C}$ and magnetic flows $F_{A}, F_{B}$ and $F_{C}$ - formed on the bases of three-phase primary currents of controlling and measuring from electric nets and transforming on the bases of detectors - a flat measure windings to secondary voltage of electromagnetic transducer.

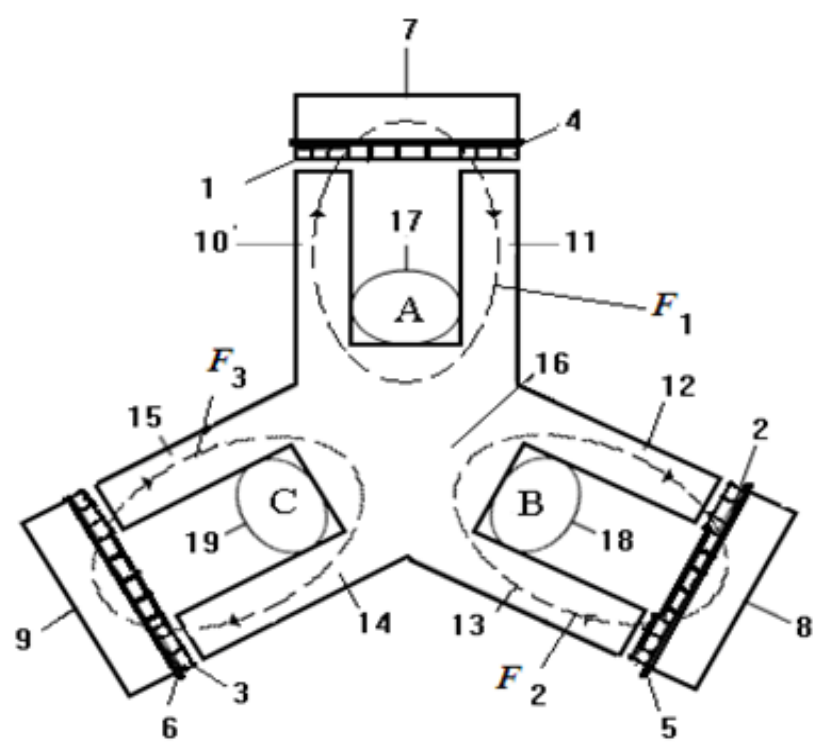

1, 2, and 3 - flat measure windings, 4, 5 and 6 - insulation platens, 7, 8 and 9 - additionally Cores, 10 and 11,12 and 13,14 and 15 - pairs if parallel Cores, 16- basically Core, 17, 18 and 19- primary windings on electrical nets

Figure 1. The constructions of Electromagnetic transducer of primary currents to secondary voltage with flat measure winding and three additionalyl magnetic Cores

Magnetic flows in magnetically systems of transudes determined as shown next:

$$
\begin{aligned}
& \mathrm{F}_{\mathrm{A}}=\mathrm{F}_{\text {макс } \mathrm{A}}\left[\mathrm{e}^{-(\mathrm{R} t / \mathrm{L})}-\cos (\omega \mathrm{t})\right] \pm \mathrm{F}_{\mathrm{rem} \mathrm{A}} \mathrm{e}_{\mathrm{I}}^{-(\mathrm{R} t / \mathrm{L})},
\end{aligned}
$$

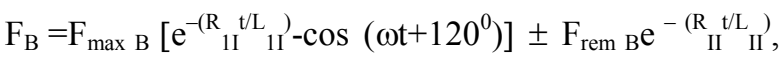

$$
\begin{aligned}
& \left.\mathrm{F}_{\mathrm{C}}=\mathrm{F}_{\max C}\left[\mathrm{e}^{-\left(\mathrm{R}_{1 I I}{ }^{\mathrm{t} / \mathrm{L}} 1{ }_{1 I I}\right)}-\cos \left(\omega \mathrm{t}-120^{\circ}\right)\right] \pm \mathrm{F}_{\text {rem C }} \mathrm{e}^{-\left(\mathrm{R}_{\text {III }} \mathrm{t} / \mathrm{L}\right.}{ }_{\text {III }}\right) \text {. }
\end{aligned}
$$

The Excess of instant importance magnetic flows in connecting mode enabling for electric loads bring on the bases of flowed from primary windings of the electromagnetic transducer of the current to voltage with flat measure winding (Figure 2 ). 


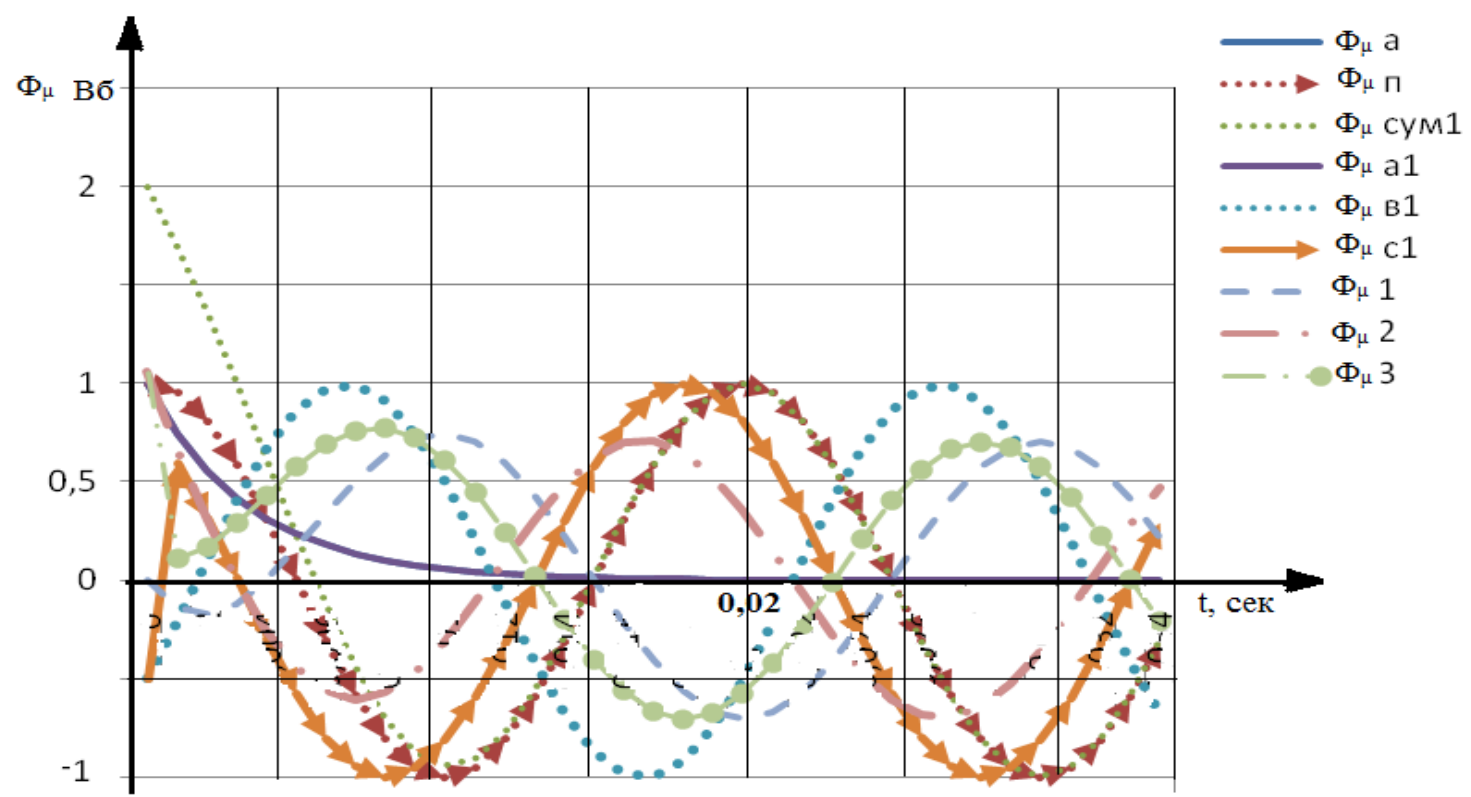

Figure 2. Graphics of the magnetic flows in magnetic systems of the electromagnetic transducers of current to voltage with flat measure winding

As shown from results of research, transitive processes in magnetic systems of the electromagnetic transducers of the current to voltage with flat measure winding is set up during $0,044 \mathrm{~s}$. after turn on of three-phase electric load to electric net of power system.

\section{Conclusions}

In the article given theoretical and experimental materials of analysis and research of output value and parameters of electromagnetic transducers of current to voltage with flat measuring winding for combined control of three-phases currents of electric nets of power systems, based on research, design a principle,; a problem of development and construction efficient electromagnetic transducers one- and polishes primary currents for secondary voltage.

1. Motivated, that the uses of the flat measuring windings in electromagnetic transducers of current to voltage as detector elements, provides receipt unified output signal with parameter: voltage $-20 \mathrm{~V}$, current $-100 \mathrm{~mA}$ and allows develop a new electromagnetic transducer of current to voltage with flat measuring windings, being up to quality combined auto control system of power of power4 systems.

2 . The Best values out voltages $-U_{\text {out }}$ are provided at value of the air clearance $\delta$ - equal $-0,002-0,003 \mathrm{~m}$ and numbers whorl of flat measuring windings $W_{F M W}$ - equal - 3 4.

3. Installed, that influence of the secondary current $I_{\text {out }}$ from flat measuring windings of transducers consist $0,017 \%$ from normalized value of primary current $I$ in . When a temperature changed surrounding ambiences, inaccuracy of the transformation increases $0,03 \%$.

Accounting entropy inaccuracy of the transducers of the current to voltage does not exceed $0,2 \%$, experimental importance of inaccuracy electromagnetic transducer of the current to voltage with flat measuring windings consist $0,21 \%$.

\section{REFERENCES}

[1] Siddikov I.Kh., Khakimov M.Kh., Anarbaev M., Bedritskiy I.M., Research of the electromagnetic transducers of the primary current to secondary voltage. Science and Education. TCSET of the II International Research and Practice Conference. Vol. I, Publishing office of «Vela Verlag Waldkraiburg», Germany, December, 18-19, 2012.

[2] Amirov S.F., Azimov R.K., Siddikov I.Kh., Khakimov M.Kh., Khushboqov B.X., Sattorov Kh.,A. Patent RUz. №04185. The transducers of no symmetry of current to voltage. // Bulleten №6. 2010. 